\title{
The Development of the Heparin Monitoring System Based on Microfluidics Technology
}

\author{
Jingwen Zhou \\ The Key School, Annapolis, MD, USA \\ Email: 1435647250@qq.com
}

How to cite this paper: Zhou, J.W. (2020) The Development of the Heparin Monitoring System Based on Microfluidics Technology. Journal of Biomaterials and Nanobiotechnology, 11, 195-213.

https://doi.org/10.4236/jbnb.2020.113013

Received: April 16, 2020

Accepted: July 10, 2020

Published: July 13, 2020

Copyright (อ 2020 by author(s) and Scientific Research Publishing Inc. This work is licensed under the Creative Commons Attribution International License (CC BY 4.0).

http://creativecommons.org/licenses/by/4.0/

\begin{abstract}
Heparin monitoring is widely used to measure the anticoagulant effect of unfractionated heparin and adjust the dose to keep within the target treatment range. This technology has applications in many fields and also prospects in the future. Its application has the advantages of rapidity, high throughput and minimum sample consumption. Many point of care devices for heparin monitoring are available. The CoaguChek device only requires a small sample size, which is obtained through a fingerstick. Over the last few years, the point-of-care (POC) testing was used widely for its convenience, efficiency, and faster turnaround times.
\end{abstract}

\section{Keywords}

Heparin Monitoring, Care Devices, Microfluidics Technology

\section{History and Development of Microfluidics}

With the development of Hagen-Poiseuille's equation, the advancement of siloxanes, and the introduction of Micro-Elecro-Mechanical Systems, the technology of Microfluidics began to grow. The invention of photolithography from the 1970s stimulated its growth even more. In 1979, S. C. Terry et al. [1] recognized a miniaturized gas chromatography on a silicon wafer, and developed the first "lab-on-a-chip" gas analysis system, mainly made of Polydimethylsiloxane (PDMS) materials.

Photolithography and chemical etching techniques helped to reduce its size by approximately three orders of magnitude compared to traditional devices. The chromatography system was formed by a sample injection valve and a long separation capillary. In the following years, several silicon-based analysis systems had been presented. In 1990, A. Manz et al. [2] introduced the concept of a miniaturized "total chemical analysis system" (micro TAS) based on the develop- 
ment of more portable analysis systems and more comprehensive analytical performance. The theoretical performances of such systems based on flow injection analysis, chromatography and electrophoresis, are compared with those of existing chemical sensors and analysis systems. In 2000, digital microfluidics was introduced by R. Fair et al. [3], which was a microactuator for the fast operation of discrete droplets (Figure 1) based on electrowetting. With two separate layers of electrodes on glass, the microactuator was realized by directly controlling surface tension. A prototype device was built and tested, which was made of a linear array of seven electrodes at a $1.5 \mathrm{~mm}$ pitch. Droplets of $100 \mathrm{mM} \mathrm{KCl}$ solution were successfully transferred between adjacent electrodes at voltages of 40 $80 \mathrm{~V}$. Repeatable transports of droplets at electrode switching rates of up to 20 $\mathrm{Hz}$ and average velocities of $30 \mathrm{~mm} / \mathrm{s}$ have been demonstrated. This speed increased by almost 100 fold compared to former electrical techniques of transporting droplets. Therefore this system was flexible and efficient, and was capable of completing complex and highly parallel microfluidic processing.

In 2007, G. Whitesides et al. [4] introduced the paper-based microfluidics, a simple method for patterning paper to create well-defined, millimeter-sized channels, comprising hydrophilic paper bounded by hydrophobic polymer (Figure 2). The pattern paper was not only low cost, but also portable. This capability was demonstrated by the simultaneous detection of glucose and protein in $5 \mathrm{~mL}$ of urine. The system was small, disposable, easy to use, and did not require external equipment, reagents or power supply. The potential applications of this paper-based microfluidics include quick and on-site diagnosis when medical resources are limited.

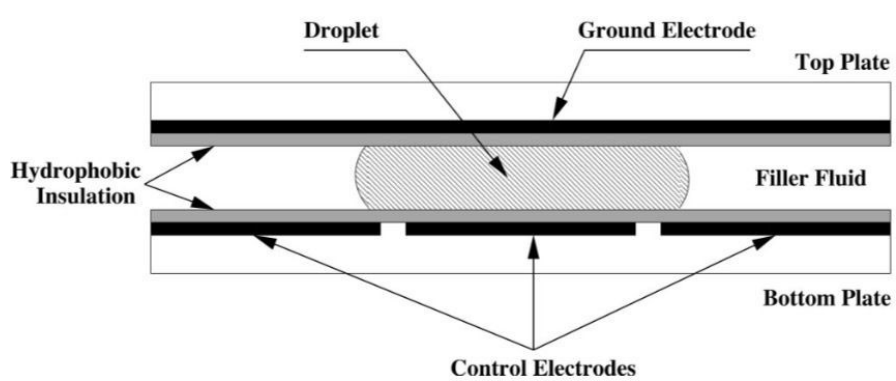

Figure 1. Schematic cross-section of the electrowetting microactuator [3].

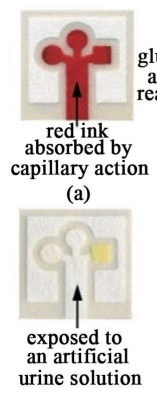

(c)

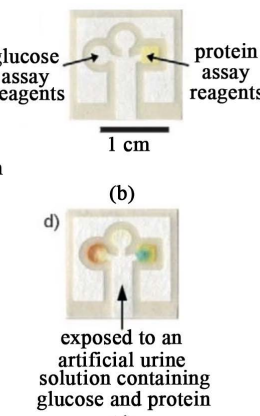

(d)

\begin{tabular}{|c|c|c|}
\hline $\begin{array}{c}\text { [glucose]/ } \\
\mathrm{mM}\end{array}$ & & $\begin{array}{c}{[\mathrm{BSA}] /} \\
\mu \mathrm{M}\end{array}$ \\
\hline 0 & & 0 \\
\hline 2.5 & & 0.38 \\
\hline 5.0 & & 0.75 \\
\hline 10 & & 1.5 \\
\hline 50 & & 7.5 \\
\hline 500 & & 75 \\
\hline
\end{tabular}

(e)

Figure 2. Chromatography paper patterned with photoresist [4]. 


\section{The Liquid Manipulation of Channel Microfluidics and Digital Microfluidics}

The simplest way to move fluids in channel Microfluidics is to use its gravity, but this method is highly unstable and inaccurate, and has many limitations. Therefore, a pressure controller was invented first, which functions by applying pressure onto the test tubes which are connected to the microfluidics chip. Later on, more accurate liquid control and more complicated functions were realized by the development of microvalves and micropumps. Over the years, many types of microvalves and micropumps have been developed to use on microfluidics systems. This paper will discuss four most basic formats of microvalves and micropumps, including the Quake microvalve, the "Doormat" valves, the Plunger microvalve, and lateral deflection valve.

In 2000, Marc A. Unger et al. [5] presented their research on the Quake microvalve, one ofmany types of microvalves. The Quake microvalve included a flow channel and several control channels placed on top of the flow channel and perpendicular to the direction of the flow channel (Figure 3). The liquid goes in the flow channel at the bottom, and the upper control channel functions as an air network. Therefore, as the pressures inside the control channels increase or decrease, the upper layer control channels can deform in order to control the fluids inside the flow channel. As air pressure increases, the channel deforms, pressing the bottom channel to stop the flow. As the pressure is eliminated, the fluids in the bottom channel resumes movement. Thanks to the development of polydimethylsiloxane (PDMS), the deformation was easily achieved. PDMS is a soft material that can be deformed by pressure.

In 2005, Nianzhen Li et al. 6 demonstrated another type of microvalve, "Doormat" valves (Figure 4). At its rest state, the fluids in the flow channel are blocked by a PDMS barrier positioned on top of it, which is a major difference from the Quake microvalve. As pressure is applied in the air network, the wall between the two networks deform, allowing liquid to go through. Since the microvalve does not require energy input to remain closed, the fluid can be stored in a highly portable manner for several days, which is an advantage compared to the Quake microvalve, of which the rest state is the flowing state.

Ju Yeoul Baek et al. [7] presented another PDMS-based microvalve called the Plunger microvalve, a multilayer design, which is an innovation compared to the previous microvalves (Figure 5).

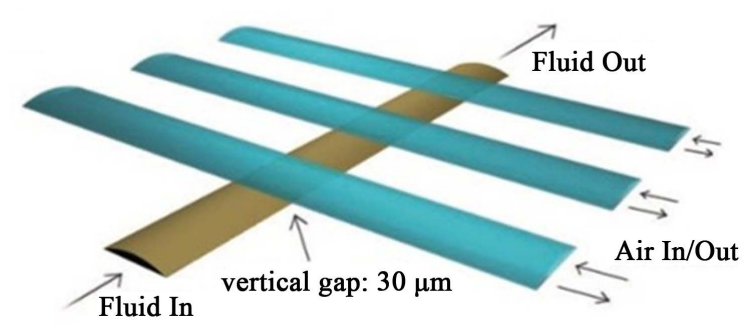

Figure 3. PDMS quake valves 5. 
(a)
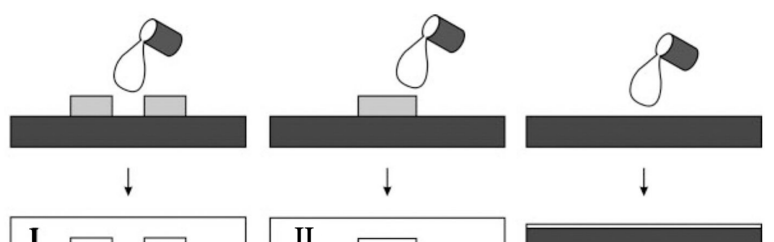

(b)

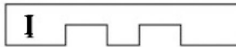

(e)

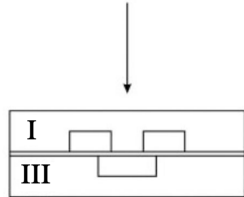

(c)

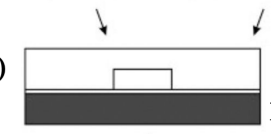

(d)

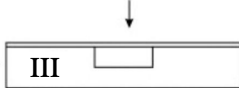

SU-8

photoresist

Si wafer

PDMS

Figure 4. "Doormat" microvalves [6].

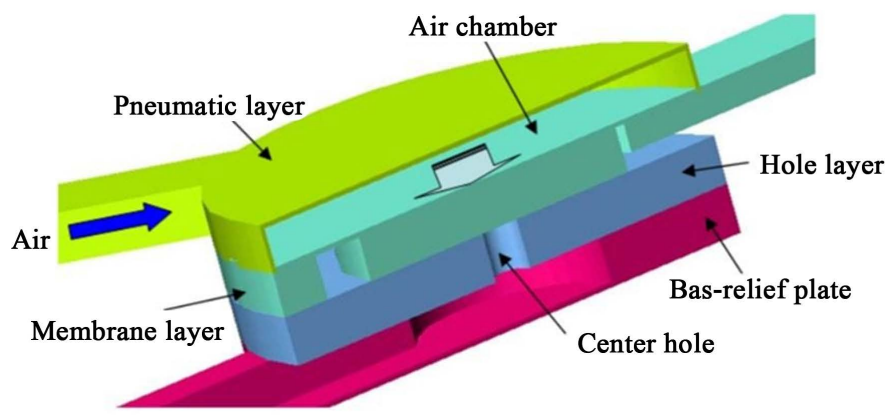

(a)

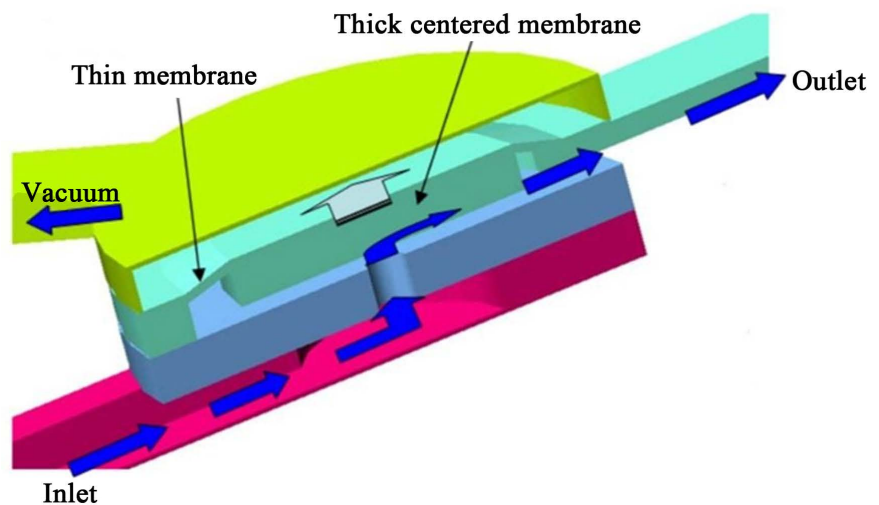

(b)

Figure 5. Schematic and operation of the thick centered valve. (a) Closing of the valve by compressed air; (b) Opening of the valve by vacuum [7].

The valve includes a thick central membrane to adjust the "on" and "off" of the flow, enabling or disabling fluid flow through the control layer at the bottom. The flow rate can be adjusted on a scale of a few microliters per minute by adjusting the pressure applied to the inlet channel. As the pressure goes higher, the fluid flow is turned on due to the deformation of the membrane layer.

In 2005, Narayan Sundararajan et al. [8] demonstrated another type of microvalve, the lateral deflection valve (Figure 6). This valve is different from the previous ones in that it has only one PDMS layer, and the fluid flow is activated by the lateral deflection of PDMS material. 


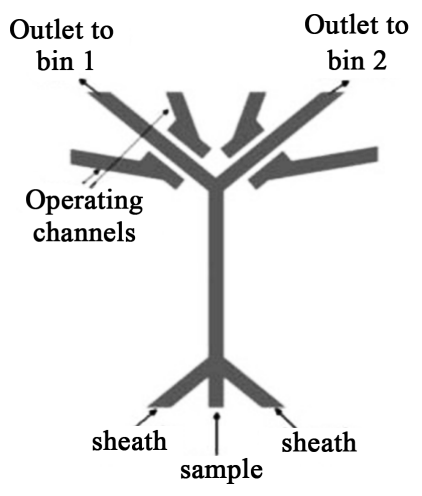

Figure 6. Schematic of lateral deflection microvalve [8].

Besides microvalves and micropumps, Frieder Mugele et al. [9] presented their research on another important technology of liquid manipulation, digital microfluidics (Figure 7). It is based on the sandwich structure with a liquid droplet between two layers of electrodes. The top plate is coated with continuous grounding electrodes, and the bottom plate contains array electrodes which can be controlled separately. When a voltage is applied, the aqueous droplets will spread on the bottom surface. Droplets are moved by turning the voltage on and off in succession across adjacent electrodes. By changing the potential along with the electrode line array, electrowetting can be used to move the droplets along the electrode array.

\section{Separation and Detection Technology}

One of the first separation methods used with microfluidics is Capillary electrophoresis, a technique that uses an electric current to separate charged particles through a capillary tube. Each end of the capillary is in a buffer, which is used to carry research molecules and neutralize negatively charged capillary walls. Pamme et al. [10] introduced the method of magnetism separation in the format of two different kinds of $\mathrm{H}$-shaped channels (Figure 8). It is now used in a variety of microfluidic applications, especially in the area of bioassays.

In 2015, Akbar et al. [11] introduced a miniaturized gas chromatography system $(\mu \mathrm{GC})$ to separate the different components in the sample. This system has the potential of achieving rapid analysis of volatile organic compounds (VOCs) on a very compact and low-power platform (Figure 9). The sample solution is injected into the instrument, and the gas flow transports the sample to the separation column, in which various components are separated. The microfabrication technology has been used here to demonstrate the single chip integration of the key components of a $\mu \mathrm{GC}$ system in a two-step planar fabrication process.

In 2006, Lazar et al. [12] suggested a microfluidic liquid chromatography (LC) system (Figure 10), which integrated separation and detection modules in one system. Liquid chromatography is more suitable for proteomic applications and biomarker screening applications because samples of complex biological components cannot be gasified. 


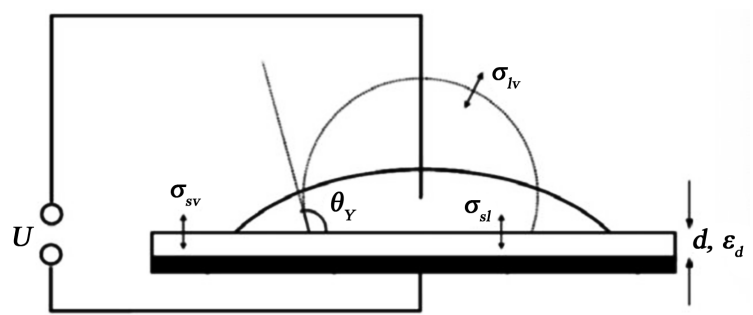

Figure 7. Digital microfluidics setup [9].

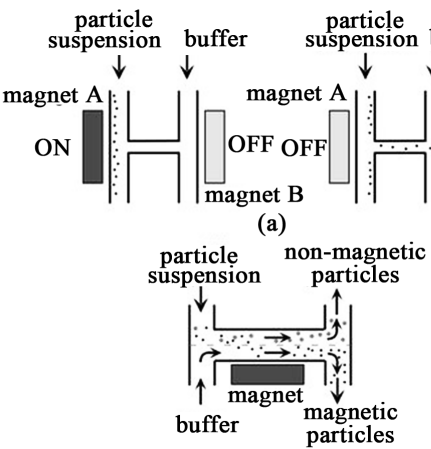

(b)

Figure 8. Principle of $\mathrm{H}$-shaped separators: (a) for particle isolation into separate streams and (b) for continuous flow separation. Redrawn with permission.

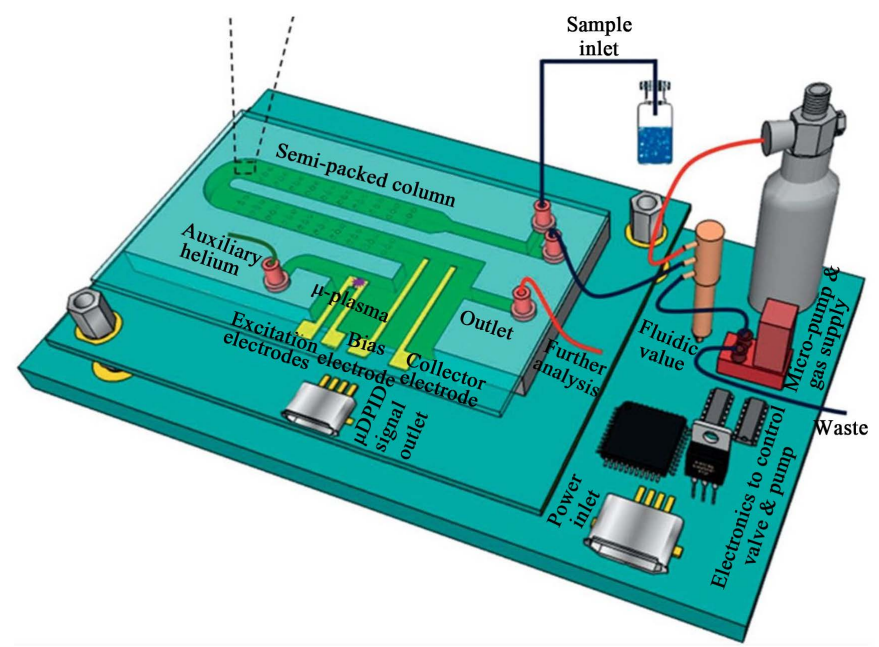

Figure 9. Setup showing the configuration of fluidic interconnections between the chip, valve, micropump, and carrier gas [11].
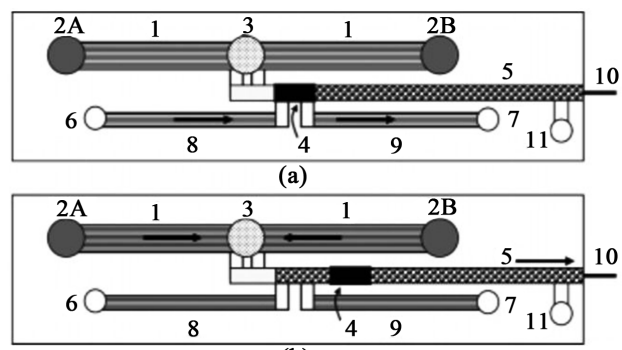

(b)

Figure 10. Schematic representation of a microfluidic LC system [12]. 
Optical detection techniques are predominantly used with digital microfluidics systems, including absorbance, fluorescence, and luminescence detection. Traditional absorbance detection is done by putting the solution of analyte into a sample cuvette. The absorbance reader then determines the absorbance by sending a known intensity of light to the sample and detecting the intensity after passing through the sample. This measurement used the Beer-Lambert law which stated that absorbance equals the molar absorption coefficient times the path length times the concentration $(\mathrm{A}=\varepsilon \mathrm{c} c)$. Fluorescence detection is also widely used, mainly for measuring compounds in solution. In fluorescence detection, a beam passes through a solution in a cuvette and the light that is emitted by the sample is measured from an angle, in order to minimize the risk of reflected incident light reaching the detector.

\section{Microfluidics Applications}

Microfluidics has been used in environmental sensing. I. Maguire et al. [13] presented a review on the application of centrifugal microfluidics in the field of environmental sensing in examining the multi-facets of microfluidic platforms (Figure 11). Roberto Pol et al. [14] presented research on the potentials of microfluidic lab-on-a-chip platforms in monitoring the environment through examining the uses and development of deployable remote autonomous microfluidic systems that detect pollutants in real time.

Microfluidics systems are also widely used in the field of medical diagnostics due to its ability to handle small sample sizes and save valuable reagents used in the assays. For example, Catherine Rivet et al. [15] examined the developments in microfluidics for medical diagnostics and integrations with biosensors through the exploration of microfluidic technology's features. Wonhwi Na et al. [16] then introduced a new microfluidic method for the quick and accurate detection of viruses by amplifying DNAs through microfluidic multi-channels (Figure 12).

Guoliang Huang et al. [17] reported their research on the development of a cheap, effective, and accurate microfluidic chip-based portable nucleic acid analyzer designed for the purpose of parallel identification of multiple clinical pneumonia

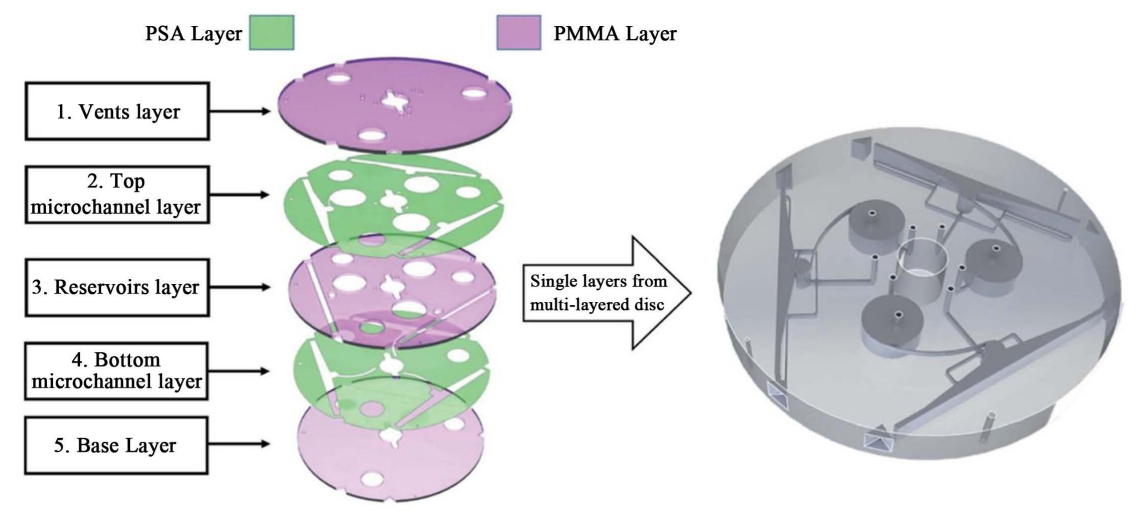

Figure 11. Microfluidics in environmental sensing [13]. 


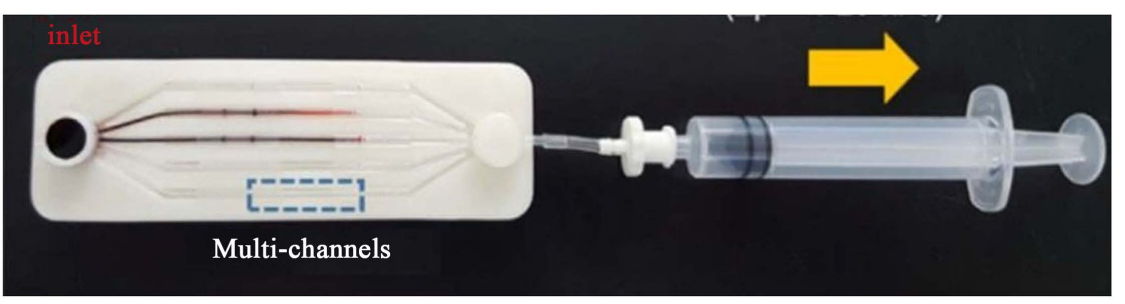

Figure 12. A photograph of the experimental apparatus [16].

related pathogens that was realized due to the microfluidic technology's flexibility for automation, integration, miniaturization, and multiplexing.

The technology of microfluidics has been employed in drug discovery as well. Amir R. Aref et al. [17] presented their research on the use of a microfluidic system to integrate tumor cell spheroids in a 3D hydrogel in close co-culture with an endothelial monolayer.

Moreover, microfluidics has been applied in microscale energy systems. For example, Hankeun Lee et al. [18] reported research on the development of a fully self-sustaining biological solar cell that bypasses common performance limitations through the use of a miniature microfluidic-based single-chambered device (Figure 13). This allowed for a reconfiguration of the anodes and cathodes as well as air-bubble trap, together resulting in the maximization of the capture of solar energy, an efficient use of material, and long-term sustainable operations (Figure 14).

In 2014, Lin Li et al. [19] demonstrated the effective potential of their optofluidic micro-photocatalytic fuel cell in simultaneously degrading organic pollutants and generating electricity. The fuel cell was realized through the incorporation of the technological combination of microfluidics and modern optics that presented key features such as fine flow control, large surface area to volume ratio and uniform light distribution. Juan Pablo Esquivel et al. [20] presented research on the integration of micro direct methanol fuel cell in a microfluidic platform that provided electrical and pumping power to a Lab-on-a-Chip system through an innovative microfluidic design that allowed specific regulation of the flow rate in the system. In 2017, J. P. Esquivel et al. [21] demonstrated their paper-based hydrogen fuel cell stand-alone power source that produced hydrogen in-situ upon the addition of liquid. This was achieved by the use of paper microfluidics technology, which could integrate microfluidic functions in a single device without adding complexity to the user.

\section{Anticoagulant Monitoring}

Coagulation is the process by which blood changes from a liquid to a gel, forming a blood clot. Fibrin, a protein in the blood vessel, works with platelets, small blood cell fragments, to form the clot. Furie et al. [22] examined thrombus formation in a living mouse, using new technologies of intravital video microscopy to visualize arterioles and venules and analyse platelets, proteins, endothelium, and the vessel wall during thrombus formation (Figure 15). A thrombus, formed 

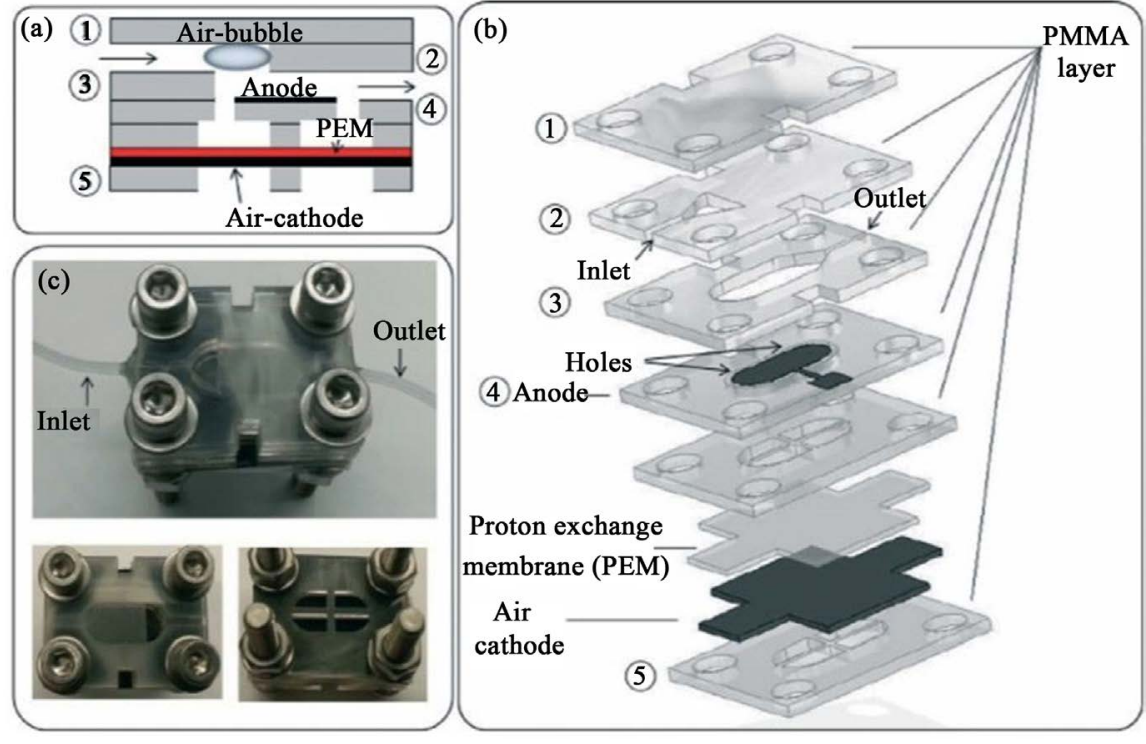

Figure 13. Biological solar cell [18].

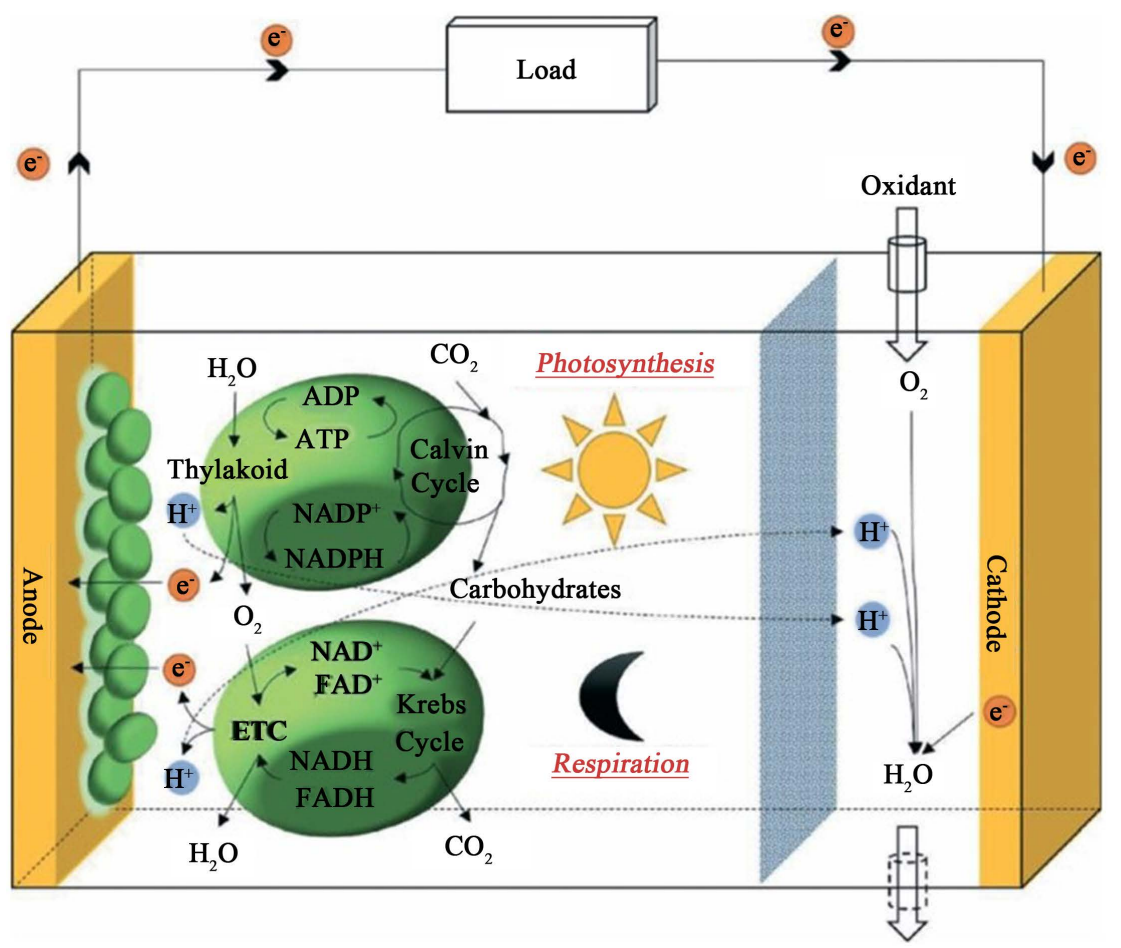

Figure 14. Principle of operation in a bio-solar cell [18].

through coagulation, is a healthy response to injury and is designed to prevent bleeding, but when thrombus blocks blood from passing through healthy blood vessels, it can be harmful. Disease regarding coagulation includes Hemophilia, a bleeding disorder in which blood does not clot normally, other clotting factor deficiencies, and platelet disorders.

Anticoagulant therapy is among one of the most widely used therapy in clinical settings. It is ordered to prevent the formation of clots in Atrial fibrillation 

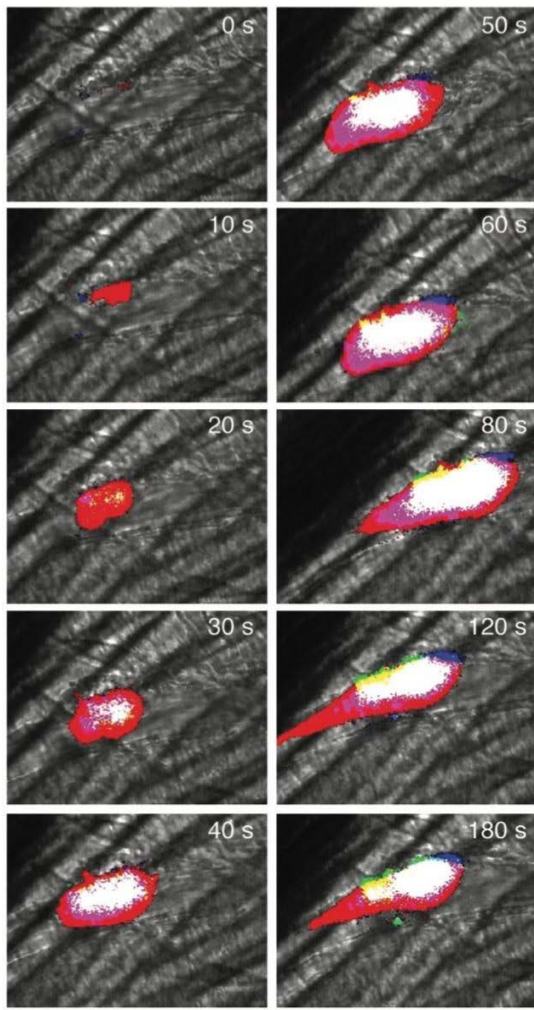

Figure 15. Birth of a thrombus [22].

(AFib), Coronary artery disease (CAD), Deep vein thrombosis, Ischemic stroke during operation, and also to reduce progression of thromboembolic disease. Various kinds of anticoagulant include heparin, warfarin, and apixaban. In 2012, Gerald A. Soff [23] presented research on a new generation of oral direct anticoagulants, which have been examined through the past five years of clinical trials. Their study focused on three drugs, either recently approved by the US Food and Drug Administration or with the most mature phase III clinical data. Jack Hirsh et al. [24] demonstrated two types of heparin, which is an anticoagulant that prevents the formation of blood clots. They introduced unfractionated heparin (UFH), a mixture of glycosaminoglycans, which catalyzes the inactivation of thrombin and other coagulation factors. UFH also binds endothelial cells, platelet factor 4 and platelets, leading to unpredictable pharmacodynamic properties. It is used for prophylaxis and treatment of venous thrombosis disorders, treatment of pulmonary embolism, and the diagnosis of acute and chronic consumptive coagulopathies. It is also used to prevent activation of the coagulation mechanism during arterial and cardiac surgery and as blood passes through an extracorporeal circuit in dialysis procedures. This paper will mainly focus on the monitoring of UFH.

Currently, several tests are available in clinical settings for heparin monitoring. In 1998, John D. Olson et al. [25] presented one of the tests, activated partial thromboplastin time (aPTT), which is a screening test used to determine the degree of anticoagulation with UFH. It measures the number of seconds it takes for 
a clot to form in a blood sample after the substance is added and helps to assess a person's ability to form blood clots. Antifactor Xa method is also used to monitor heparin level. In the monitoring of heparin level using the anti-fXa method, the patient's plasma is mixed with excess amount FXa which is quantified to form an AT-heparin-Xa Complex, leaving residual excess Factor Xa. A chromogenic substrate is then added, and the residual Factor Xa produces an optical readout that can be measured by common laboratory equipment.

\section{Automated Lab Testing and the Point-of-Care Devices for Heparin Monitoring}

This section will discuss automated lab testing instruments and point-of-care devices for Heparin monitoring.

Mechanical detection and optical detection are the two most commonly used detection methods for automatic lab testing instruments. According to Steve Kitchen et al. [26], this method is based on the detection of an increase in plasma viscosity when fibrin is formed. An electromagnetic field is generated and applied to sample cuvettes to detect the movement of a stainless steel sphere placed inside the sample. The steel sphere swings from one side to the other in a plasma reagent solution. As the fibrin begins to form, viscosity increases and the sphere's movement is delayed. When the sphere's oscillation movement reaches a predetermined level, the chronometer stops, indicating the time of plasma coagulation (Figure 16). The commercial instruments using mechanical detection include ACL Elite Pro, ACL AcuStar, and Cascade M-4.

Optical detection is another testing method. Optical-based detection is used by most central laboratory analyzers due to its robustness and well-established means of performing bioassays. Photo-optical is one of the four categories of optical detection. Optical systems are based on the notion that clot formation induces change in the plasma's optical density. As the clot is formed, there are changes in the optical characteristics from the initial reading of the plasma. These changes are monitored and used to derive the time taken for a particular degree of change to occur [28].

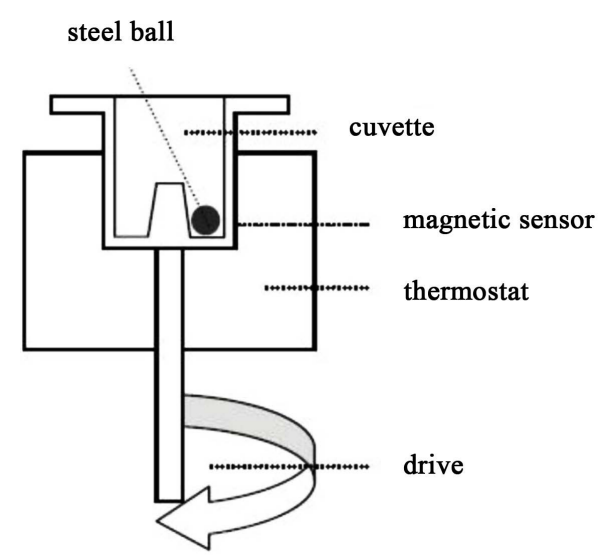

Figure 16. Patented ball technology [27]. 
The point-of-care (POC) testing is used more widely for its convenience, efficiency, and faster turnaround times. Lode [28] suggested that significant advances in assay and detection technologies have recently facilitated the introduction of truly quantitative, sophisticated immunoassay methods to POC settings, with the analytical performance characteristics approaching those of conventional laboratory assays. People can use POC in the home, the workplace, the doctor's surgery, the pharmacy, as well as in the hospital. Some Point of Care Testing instruments mentioned in this paper are listed in Table 1.

Andrew et al. [29] acknowledged that PoCT technologies can be split into two categories. Of the two major categories the first is small handheld devices, providing qualitative or quantitative determination of an increasing range of analytes. The dominant technologies here are glucose biosensor strips and lateral flow strips using immobilised antibodies to determine a range of parameters including cardiac markers and infectious pathogens. The second category of devices is larger, often bench-top devices which are essentially laboratory instruments which have been reduced in both size and complexity. These include critical care analysers and, more recently, small haematology and immunology analysers. CoaguChek XS Plus System is based on light scattering, one of the categories of optical detection method (Figure 17). It is used for warfarin management of patients at the point of care. When using this instrument, only a small sample size is required, which is obtained through a fingerstick.

In 2002, Jaryno et al. [30] presented another testing instrument, called Hemochron ${ }^{\otimes}$ Signature Elite system which uses optical-mechanical detection. In some POC devices both optical and mechanical detection technologies are combined, and Hemochron ${ }^{\circledR}$ is one of them. It is a third generation point-of-care (POC) whole blood coagulation analyzer that retains the clinical utility of the Hemochron standard (801/8000) while providing a data management program that assists the POC coordinator with Quality Assurance (QA) compliance.

In 1992, Jian-Sheng Wang et al. presented another testing instrument using optical-mechanical, Hepcon Hemostasis Management System (HMS). HMS is based on the measurement and maintenance of individually calculated target

Table 1. Some point of care testing instruments.

\begin{tabular}{ccc}
\hline Name & Company & Detection Method \\
\hline CoaguChek XS Plus System & Roche Diagnostics & Light Scattering \\
Hemochron Signature Elite System & ITC & Optical-Mechanical \\
Hepcon Hemostasis Management System & Medtronic Hemotec & Optical-Mechanical \\
Rapidpoint Coag Machine & Bayer & Paramagnetic-Optical \\
Thrombotrack ${ }^{\mathrm{TM}}$ Solo & Axis-Shield & Mechanical Detection \\
The ACT Plus System & Medtronic & Electromechanical \\
Actalyke ${ }^{\circledast}$ technology & Helena Laboratories & Electromagnetic \\
i-STAT ACT & Abbott & Electrochemical
\end{tabular}




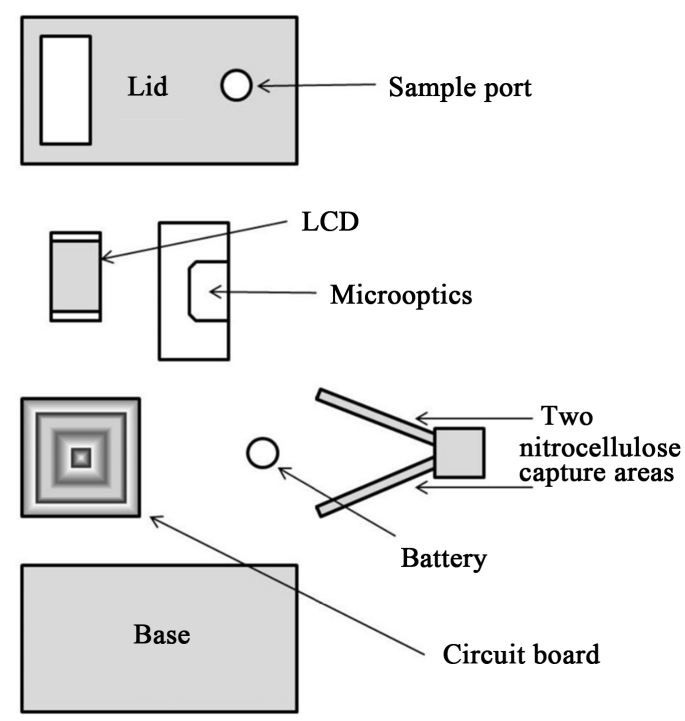

Figure 17. Schematic of microfluidic cassette enclosed in a reader containing electronics and microoptical detectors [27].

heparin levels. Blood specimens (400 microL) are inserted in each of the two wells of a cartridge. This machine uses a mechanical plunger-flag assembly that is dipped in and out of activated blood samples in the cartridge. The machine optically senses the time that it takes to move the plunger through the blood specimen in the cartridge and the presence of clot is based on the detection of a decreased rate of drop of the plunger flag assembly: clotting time is defined as the time at which a certain "drop time" threshold for the plunger is reached.

In 2001, Prisco et al. [29] presented Rapidpoint Coag machine, a testing instrument using Paramagnetic-Optical. This machine uses disposable test cards containing a reaction chamber with test-specific reagents (celite and stabilizers) and paramagnetic iron oxide particles (PIOPs) which move in response to an oscillating magnetic field. When 30 -microL blood is added to the reaction chamber it dissolves the dry reagent. The occurrence of coagulation results in a slowing and cessation of PIOPs movement. This technology is based on infrared sensing of PIOPs motion, which causes a change in light transmission.

In 2013, Harris et al. [31] presented Thrombotrack ${ }^{\mathrm{TM}}$ Solo, a machine using mechanical detection. It is the most widely used and traditional method of clot detection. A sensor is used to detect the movement of a ball in the bottom of the cuvette. When clot formation happens in the cuvette the movement of the ball is impeded and it becomes lodged at the edge of the cuvette.

In 2016, Joshi et al. [32] presented Actalyke ${ }^{\oplus}$ technology, a machine using electromagnetic detection. This detection method is based on a two-point detection mechanism. One magnet is positioned at $0^{\circ}$ while the other one is at $90^{\circ}$. The early detection of fibrin can be obtained when the magnet rotates through these positions. Electrochemical is another detection method in POC testing, presented by Harris et al. [27] This detects the transfer of charge from an electrode to a solid or liquid phase (Figure 18). 


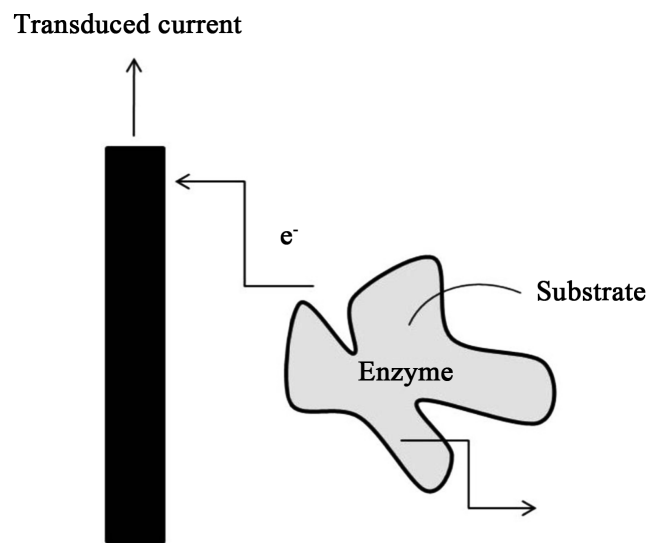

Figure 18. Direct electron transfer between an electrode and an enzyme molecule [27].

In 2011, Elizabeth et al. [33] suggested i-STAT ${ }^{\circledast}$ ACT, a machine based on electrochemical detection. It is used in vitro diagnostic test that uses fresh, whole blood, and is used to monitor high-dose heparin anticoagulation frequently associated with cardiovascular surgery.

\section{Looking into the Future: Novel Technology of POC for Heparin Monitoring}

The novel technology of point-of-care devices based on microfluidics systems for Heparin monitoring is now under fast development.

In 2011, Magdalena M. Dudek et al. [34] reported work on microstructured lateral flow platforms used in combination with fluorescently labelled fibrinogen to detect microscopic clot formation that compliments the inherent variability and imprecision of microfluidic blood coagulation devices. They reported development on a clotting time measurement assay which uses a fluorescent probe to precisely detect the onset of fibrin clot formation using a microstructured surface that enhances the formation of multiple localized clot loci and results in the abrupt redistribution of the fluorescent label at the onset of clot formation in both whole blood and plasma (Figure 19).

Marco Ranucci et al. [35] in 2014 investigated the viscosity changes of whole blood in select subjects with a normal coagulation profile using a cone-on-plate viscometer to explore the changes in blood viscosity at different shear rates, taking into account research on Microfluidic thrombosis under multiple shear rates and antiplatelet therapy doses and biomicrofluidics technology.

Two years later, Abhishek Jain [36] describe a microfluidic device that mimics a network of stenosed arteriolar vessels, permitting evaluation of blood clotting within small sample volumes under pathophysiological flow that compliment the downsides of current monitoring devices which do not measure effects of hemodynamic forces that contribute significantly to platelet function and thrombus formation (Figure 20).

Later on in 2017, Michael A. Hegener et al. [37] presented the results of clinical experience using a paper-based lateral flow diagnostic device based on microfluidic 


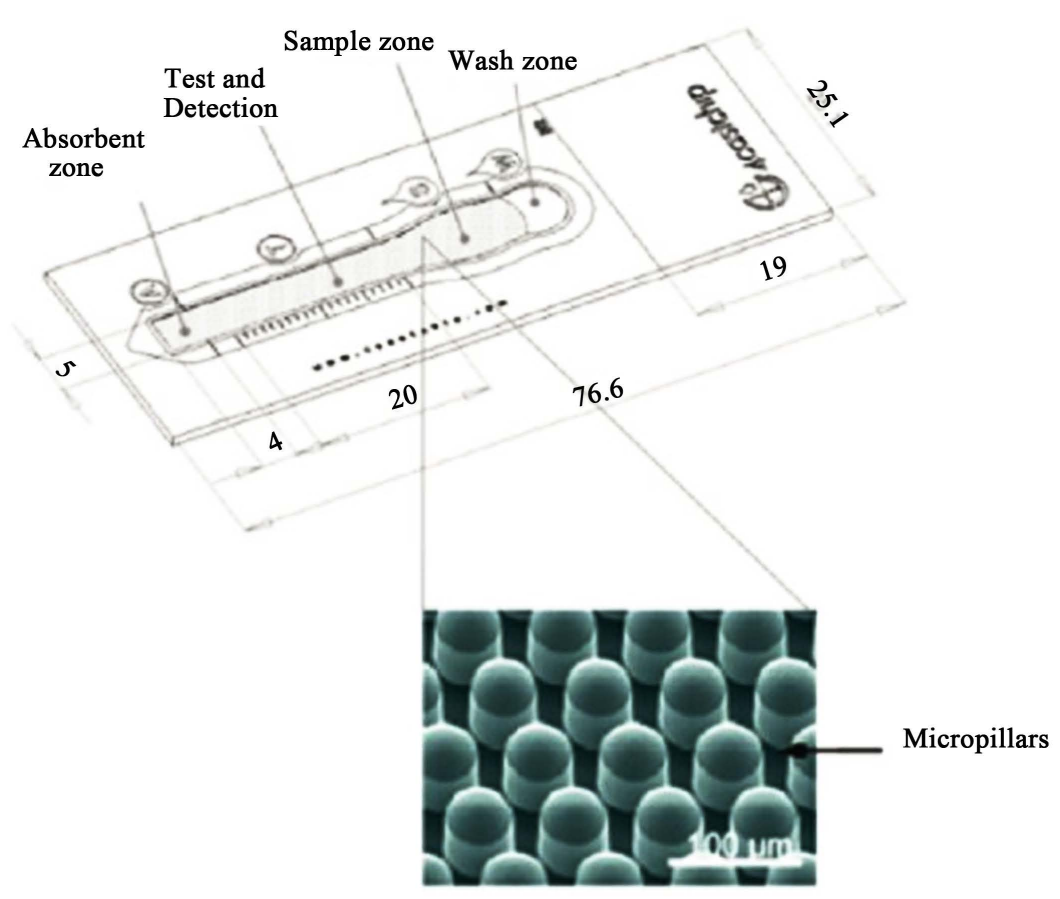

Figure 19. Graphical representation of the B 2.2 micropillar lateral flow device [34].

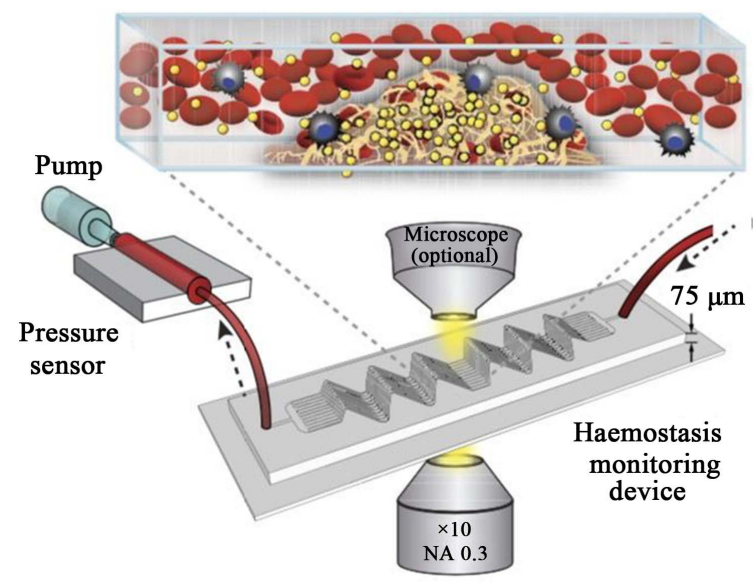

Figure 20. Schematic of the haemostasis monitor device and method [36].

technology to aid point-of-care coagulation monitoring, from which they predicted that this low-cost device holds great potential for clinical use in identifying anticoagulated patients taking vitamin $\mathrm{K}$ antagonists who are outside of the desired therapeutic efficacy window (Figure 21).

In 2018, Leanne F. Harris and Anthony J. Killard [38] reviewed the development of the use of microfluidic device development in the field of hemostasis and coagulation biology on the bases that microfluidic devices can identify a wide range of diagnostic targets and perform better than traditional testing. Microfluidic research in the area of commercial miniaturized point-of-care testing devices has been focused on the measurement of platelet activity and the identification of coagulation proteins. 


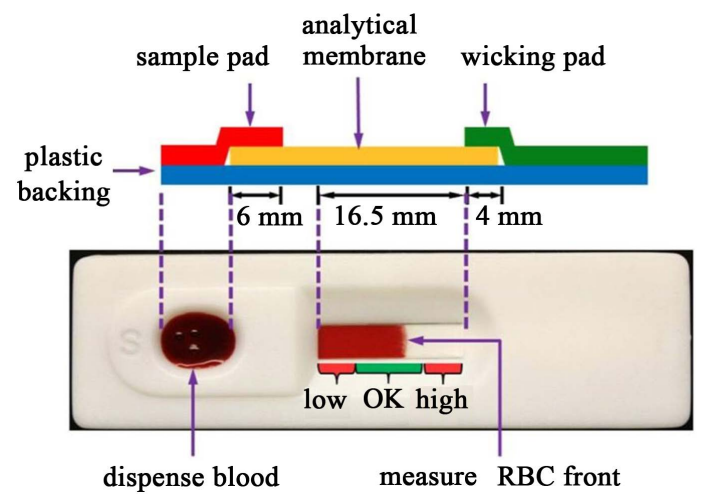

Figure 21. Schematic design and photograph of the experimental, paper-based microfluidic lateral flow assay (LFA) device [37].

In the same year, Vijay Govindarajan et al. [39] demonstrated how the growth, structure, and mechanical properties of clots under flow are shaped by the simultaneously varying tissue factor surface density and its exposure area in their research.

\section{Summary}

Heparin monitoring is widely used to measure the anticoagulant effect of unfractionated heparin and adjust the dose to keep within the target treatment range. Heparin prevents the formation of blood clots, and heparin anticoagulant therapy is crucial in the treatment and prevention of blood clots caused by certain medical conditions or medical procedures. It is also used before surgery to reduce the risk of blood clots. The technology of microfluidics is effectively used in heparin monitoring. This technology has applications in many fields and also prospects in the future. Its application has the advantages of rapidity, high throughput and minimum sample consumption, which are very important in Point of Care devices.

Tests that are commonly used in clinical settings for heparin monitoring include activated partial thromboplastin time (aPTT), and anti-factor Xa. aPTT is a screening test used to determine the degree of anticoagulation with UFH. The anti-factor Xa is an assay more commonly used for monitoring patients on LMWH therapy.

Over the last few years, the point-of-care (POC) testing was used widely for its convenience, efficiency, and faster turnaround times. People can use POC in the home, the workplace, the doctor's surgery, the pharmacy, as well as in the hospital.

Many point of care devices for heparin monitoring are available, such as the CoaguChek XS Plus System, which is based on light scattering, and the i-STAT ACT device, which uses electrochemical detection. The CoaguChek device only requires a small sample size, which is obtained through a fingerstick. In the future, POC testing should still improve to reduce the sample size it takes so that patients can be more comfortable with the test. 


\section{Conflicts of Interest}

The author declares no conflicts of interest regarding the publication of this paper.

\section{References}

[1] Terry, S.C., Jerman, J.H. and Angell, J.B. (1979) A Gas Chromatographic Air Analyzer Fabricated on a Silicon Wafer. IEEE Transactions on Electron Devices, 26, 1880-1886. https://doi.org/10.1109/T-ED.1979.19791

[2] Manz, A., Graber, N. and Widmer, H.M. (1990) Miniaturized Total Chemical Analysis Systems: A Novel Concept for Chemical Sensing. Sensors and Actuators B: Chemical, 1, 244-248. https://doi.org/10.1016/0925-4005(90)80209-I

[3] Pollack, M.G., Fair, R.B. and Shenderov, A.D. (2000) Electrowetting-Based Actuation of Liquid Drop Lets for Microfluidic Applications. Applied Physics Letters, 77, 1725-1726. https://doi.org/10.1063/1.1308534

[4] Martinez, A.W., Phillips, S.T., Butte, M.J. and Whitesides, G.M. (2007) Patterned Paper as a Platform for Inexpensive, Low-Volume, Portable Bioassays. Angewandte Chemie International Edition, 46, 1318-1320.

https://doi.org/10.1002/anie.200603817

[5] Unger, M.A., Chou, H.P., Thorsen, T., Scherer, A. and Quake, S.R. (2000) Monolithic Microfabricated Valves and Pumps by Multilayer Soft Lithography. Science, 288, 113-116. https://doi.org/10.1126/science.288.5463.113

[6] Li, N., Hsu, C.-H. and Folch, A. (2005) Parallel Mixing of Photolithographically Defined Nanoliter Volumes Using Elastomeric Microvalve Arrays. Electrophoresis, 26, 3758-3764. https://doi.org/10.1002/elps.200500171

[7] Baek, J.Y., Park, J.Y., Ju, J.I., Lee, T.S. and Lee, S.H. (2005) A Pneumatically Controllable Flexible and Polymeric Microfluidic Valve Fabricated via in Situ Development. Journal of Micromechanics Microengineering, 15, 1015-1020. https://doi.org/10.1088/0960-1317/15/5/017

[8] Sundararajan, N., Kim, D. and Berlin, A.A. (2005) Microfluidic Operations Using Deformable Polymer Membranes Fabricated by Single Layer Soft Lithography. Lab on a Chip, 5, 350-354. https://doi.org/10.1039/b500792p

[9] Mugele, F. and Baret, J.-C. (2005) Electrowetting: From Basics to Applications. Journal of Physics. Condensed Matter, 17, R705-R774. https://doi.org/10.1088/0953-8984/17/28/R01

[10] Pamme, N., Eijkel, J.C.T. and Manz, A. (2006) On-Chip Free-Flow Magnetophoresis: Separation and Detection of Mixtures of Magnetic Particles in Continuous Flow. Journal of Magnetism and Magnetic Materials, 307, 237-244. https://doi.org/10.1016/j.jmmm.2006.04.008

[11] Akbar, M., Restaino, M. and Agah, M. (2015) Chip-Scale Gas Chromatography: From Injection through Detection. Microsystems \& Nanoengineering, 1, Article No. 15039. https://doi.org/10.1038/micronano.2015.39

[12] Lazar, I.M., Trisiripisal, P. and Sarvaiya, H.A. (2006) Microfluidic Liquid Chromatography System for Proteomic Applications and Biomarker Screening. Analytical Chemistry, 78, 5513-5524. https://doi.org/10.1021/ac060434y

[13] Maguire, I., O’Kennedy, R., Ducrée, J. and Regan, F. (2018) A Review of Centrifugal Microfluidics in Environmental Monitoring. Analytical Methods, 10, 1497-1515. https://doi.org/10.1039/C8AY00361K 
[14] Pol, R., Céspedes, F., Gabriel, D. and Baeza, M. (2017) Microfluidic Lab-on-a-Chip Platforms for Environmental Monitoring. TrAC Trends in Analytical Chemistry, 95, 62-68. https://doi.org/10.1016/j.trac.2017.08.001

[15] Rivet, C., Lee, H., Hirsch, A., Hamilton, S. and Lu, H. (2011) Microfluidics for Medical Diagnostics and Biosensors. Chemical Engineering Science, 66, 1490-1507. https://doi.org/10.1016/j.ces.2010.08.015

[16] Na, W., Nam, D., Lee, H. and Shin, S. (2018) Rapid Molecular Diagnosis of Infectious Viruses in Microfluidics Using DNA Hydrogel Formation. Biosensors and Bioelectronics, 108, 9-13. https://doi.org/10.1016/j.bios.2018.02.040

[17] Aref, A.R., et al. (2013) Screening Therapeutic EMT Blocking Agents in a ThreeDimensional Microenvironment. Integrative Biology, 5, 381-389. https://doi.org/10.1039/C2IB20209C

[18] Lee, H. and Choi, S. (2015) A Micro-Sized Bio-Solar Cell for Self-Sustaining Power Generation. Lab on a Chip, 15, 391-398. https://doi.org/10.1039/C4LC01069H

[19] Li, L., et al. (2014) Optofluidics Based Micro-Photocatalytic Fuel Cell for Efficient Wastewater Treatment and Electricity Generation. Lab on a Chip, 14, 3368-3375. https://doi.org/10.1039/C4LC00595C

[20] Esquivel, J.P., et al. (2012) Fuel Cell-Powered Microfluidic Platform for Lab-on-a-Chip Applications. Lab on a Chip, 12, 74-79. https://doi.org/10.1039/C1LC20426B

[21] Esquivel, J.P., et al. (2017) Single-Use Paper-Based Hydrogen Fuel Cells for Pointof-Care Diagnostic Applications. Journal of Power Sources, 342, 442-451. https://doi.org/10.1016/j.jpowsour.2016.12.085

[22] Furie, B. (2005) Thrombus Formation in Vivo. The Journal of Clinical Investigation, 115, 3355-3362. https://doi.org/10.1172/JCI26987

[23] Soff, G.A. (2012) A New Generation of Oral Direct Anticoagulants. Arteriosclerosis, Thrombosis, and Vascular Biology, 32, 569-574. https://doi.org/10.1161/ATVBAHA.111.242834

[24] Hirsh, J. and Raschke, R. (2004) Heparin and Low-Molecular-Weight Heparin. Chest, 126, 188S-203S. https://doi.org/10.1378/chest.126.3_suppl.188S

[25] Olson, J.D., et al. (1998) College of American Pathologists Conference XXXI on Laboratory Monitoring of Anticoagulant Therapy. Archives of Pathology \& Laboratory Medicine, 122, 782-798.

[26] Kitchen, S. and McCraw, A. (2000) Diagnosis of Hemophilia and Other Bleeding Disorders. 150.

[27] Harris, L.F. and Killard, A.J. (2012) Heparin Monitoring: From Blood Tube to Microfluidic Device. In: Piyathilake, D.E. and Liang, R., Eds., Heparin, Properties, Uses and Side Effects, Nova Science Publishers, Hauppauge, NY.

[28] Von Lode, P. (2005) Point-of-Care Immunotesting: Approaching the Analytical Performance of Central Laboratory Methods. Clinical Biochemistry, 38, 591-606. https://doi.org/10.1016/j.clinbiochem.2005.03.008

[29] St John, A. and Price, C.P. (2014) Existing and Emerging Technologies for Point-ofCare Testing. The Clinical Biochemist Reviews, 35, 155-167.

[30] Jaryno, S., et al. (2000) Validation of a New Whole Blood Coagulation Monitoring System. The Journal of the American Society of Extra-Corporeal Technology, 34, 271-275.

[31] Harris, L.F., Castro-López, V. and Killard, A.J. (2013) Coagulation Monitoring Devices: Past, Present, and Future at the Point of Care. TrAC Trends in Analytical Chemistry, 50, 85-95. https://doi.org/10.1016/j.trac.2013.05.009 
[32] Joshi, K. H., et al. (2016) Detection of Heparin Level in Blood Using Electromagnetic Wave Spectroscopy. 2016 9th International Conference on Developments in eSystems Engineering (DeSE), Liverpool, 31 August-2 September 2016, 329-334. https://doi.org/10.1109/DeSE.2016.51

[33] Lewandrowski, E.L., et al. (2011) Clinical Evaluation of the i-STATKaolin Activated Clotting Time (ACT) Test in the Different Clinical Settings in a Large Academic Urban Medical Center: Comparison with the Medtronic ACT Plus. American Journal of Clinical Pathology, 135, 741-748. https://doi.org/10.1309/AJCPSF8ASGONNQM6

[34] Dudek, M.M., Kent, N., Gustafsson, K.M., Lindahl, T.L. and Killard, A.J. (2011) Fluorescence-Based Blood Coagulation Assay Device for Measuring Activated Partial Thromboplastin Time. Analytical Chemistry, 83, 319-328. https://doi.org/10.1021/ac102436v

[35] Ranucci, M., Laddomada, T., Ranucci, M. and Baryshnikova, E. (2014) Blood Viscosity during Coagulation at Different Shear Rates. Physiological Reports, 2, e12065. https://doi.org/10.14814/phy2.12065

[36] Jain, A., et al. (2016) A Shear Gradient-Activated Microfluidic Device for Automated Monitoring of Whole blood Haemostasis and Platelet Function. Nature Communications, 7, Article No. 10176. https://doi.org/10.1038/ncomms10176

[37] Hegener, M.A., Li, H., Han, D., Steckl, A.J. and Pauletti, G.M. (2017) Point-of-Care Coagulation Monitoring: First Clinical Experience Using a Paper-Based Lateral Flow Diagnostic Device. Biomedical Microdevices, 19, Article No. 64. https://doi.org/10.1007/s10544-017-0206-Z

[38] Harris, L.F. and Killard, A.J. (2018) Microfluidics in Coagulation Monitoring Devices: A Mini Review. Analytical Methods, 10, 3714-3719. https://doi.org/10.1039/C8AY01230J

[39] Govindarajan, V., et al. (2018) Impact of Tissue Factor Localization on Blood Clot Structure and Resistance under Venous Shear. Biophysical Journal, 114, 978-991. https://doi.org/10.1016/j.bpj.2017.12.034 